\title{
A Study of the Incidence and Outcome of Foot Weakness in Leprosy
}

\author{
A. GRACE WARREN \\ and \\ JOYCE LEDGER
}

Hay Ling Chau Leprosarium, Hong Kong

\begin{abstract}
The permanency of paralysis in the foot of a patient with borderline leprosy should not be considered inevitable. Spontaneous functional recovery does occur in a proportion of patients, and the percentage of those recovering can be increased by correct medication and other therapeutic means. Careful observation of 300 patients under treatment has shown that thiambutosine as the initial drug does reduce the incidence of acute paralysis and also appears to encourage recovery of function.
\end{abstract}

Disability in leprosy is usually due to paralysis and the resultant deformity. The skin lesions will subside with adequate antileprotic therapy and, unless they are gross, do not result in much disability.

Paralysis may occur in all types of leprosy. It may be an acute process in those with high immunological response (TT-BT-BB) and slowly progressive in those with a poor immunological response (BL-LL). The BT-BB-BL group are frequently of unstable immunological type and may develop an acute paralysis at one stage and a chronic neuropathy at another time. Classification of leprosy types is according to Ridley and Jopling (1962).

It has often been suggested that once a complete paralysis has occurred it cannot be reversed, and early operation has been recommended. A review of patients under our treatment has shown that some recovery does spontaneously occur, especially in patients receiving antileprotic drug treatment and simple physiotherapy (Furness, 1972) with a support, to prevent the occurrence of contractures and the overstretching of the affected muscle and tendon as recommended by Price (1964). Where normal locomotion is not possible. this includes the use of a toe-raising spring, except when the patient is immobilized by plaster of Paris or some other splint. We have consistently used a simple home-made device for our patients (Warren, 1970).

Impressions have been gained that recovery of leg paralysis is especially common (Fritschi, 1971), and a study was undertaken to determine the incidence. The contributing factors which may influence the recovery and the interval during which recovery may occur were also investigated. 


\section{Material and Method}

The records of 200 patients selected by consecutive admission number, were examined to determine the number who had some significant degree of muscle weakness of the leg, and the final outcome of therapy. The history given of the severity and duration of the disease and any paralysis before admission is of ten unreliable, partly due to the traditional desire in this area to hide the disease. However, patients' statements have been used here for lack of other information.

The group examined were those admitted in the period October 1963 to July 1965, at which time no routine for treatment of an acute paralysis had been established, except simple physiotherapy to maintain mobility and the provision of a toe-raising spring to be worn at all times until full strength was recovered or surgery performed. Since that time, various regimes of treatment have been tried.

The 200 selected patients included 170 males and 30 females (normal ratio is 1 female to 2.6 males in this institution). The feet affected were 46 left feet and 44 right feet. Most of the patients were admitted because of positive skin smears, though 12 with a negative skin smear (BI negative) were admitted for reconstructive surgery, and 4 of these because of foot problems.

The leprosy types of the whole group (by clinical classification on admission) were:

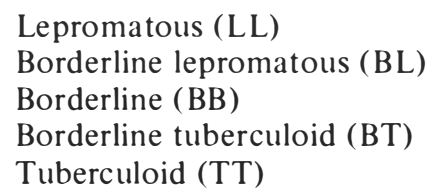
81 patients
48 patients
48 patients
16 patients
7 patients.

Most patients at that time (1963-65) were treated with dapsone or Sulphetrone initially, with routine supportive medication.

If reaction was a problem, sulphones were given in very low dosage or completely stopped, but rarely were corticosteroids used. However, all patients with any weakness or deformity of the foot were encouraged to wear suitable shoes and to use a toe-raising spring until full recovery had been present for six months or surgery was performed.

As part of routine medication, most patients received calcium lactate tablets, multivitamins (especially the B group) and iron when needed; more recently Chloroquin, Phenergan and Largactil have been used with increasing frequency, since these seem to improve the patients' general feeling of well-being and to reduce reaction. Stibophen was freely used at that time and ACTH only rarely.

In this institution, muscle assessment is according to a modification of the $0-5$ Voluntary Muscle Testing System given in the M.R.C. Report of 1943. This modification was described by Goodwin in 1968. Grading is from 1 for a muscle flicker to 5 for normal strength and range of movement. If functional movement exists a muscle will be graded 4 or 5 depending on its strength against resistance. In this article "marked paresis" implies that the tibialis anterior muscle and usually the extensors of the toes are graded 2 or less.

\section{Results}

Of the 200 patients reviewed (400 feet) it was found that:

29 feet had complete paralysis on admission

15 feet developed complete paralysis during treatment. 
44 feet had had complete paralysis at some time.

30 feet had definite muscle weakness on admission.

16 feet developed definite muscle weakness during treatment.

46 feet had definite muscile weakness at some time

In both of these groups, some patients showed recovery of muscle power. Out of the 56 patients affected, 34 had bilateral involvement, giving a total of 90 feet; these may also be divided into the following two groups:

Group 1 Patients who had marked paresis or paralysis and recovered functional power, a total of 51 feet (42 patients).

(a) Feet with acute complete loss:

(i) 3 developed paralysis before admission ( 3 patients).

(ii) 16 developed paralysis while under treatment ( 13 patients).

(b) Feet with some weakness but which adequately recovered so that surgery was not required.

(i) 17 weak on admission ( 15 patients).

(ii) 15 became weak after admission (11 patients).

Group 2 Patients advised to have surgery for foot-drop correction, a total of 39 feet (31 patients).

(a) 23 feet ( 19 patients) with complete drop undergoing foot-drop correction.

(b) 3 feet ( 3 patients) with weakness on admission who eventually had foot-drop correction surgery.

(c) 5 feet (3 patients) who had foot-drop surgery but who might not have required it if observed for a longer period.

(d) 8 feet ( 6 patients). These were patients who, though it was probably needed, did not have surgery for various reasons.

Group 1(a)(i). There were 19 feet (16 patients) in this group; 3 had paralysis on admission, 16 developed paralysis or marked paresis while under treatment. These included 2 of LL, 7 of BL, 9 of BB, 1 of BT type leprosy, in 12 males with 15 feet affected and 4 females with 4 feet affected. The age range was 17 to 53 years.

The details of the 3 patients with paralysis when treatment was started. in whom recovery occurred (Group 1(a)i) are shown in Table 1. All were given toe-raising springs.

Group I(a)(ii). The 16 feet (13 patients) which developed paralysis after the commencment of therapy can be divided into two groups. First, 9 feet ( 9 patients) in whom the acute paralysis occurred within 8 months of commencement of treatment and was usually associated with an upgrading reaction of BL to

TABLE I

Patients with paralysis on admission in whom recovery occurred

\begin{tabular}{llll}
\hline Patient's number & 1700 & 1743 & 1789 \\
Duration of paralysis on admission & 2 years & 1 year & 2 months \\
Recovery complete in & 8 months & 2 years & 1 year \\
Leprosy type & BB & BB & BB \\
Sex & M & F & M \\
Antileprotic drug & DDS & DDS & DDS \\
Special therapy & ACTH & & Left \\
Foot affected & Left & Right & L \\
\hline
\end{tabular}


BB type. These patients were usually given initially low dosage sulphone therapy, but in some patients this had been discontinued because of the severity of the reaction. In 2 patients no appreciable amount of sulphone was given before the paralysis; they were then given thiambutosine soon after the paralysis occurred. Second, a group of 7 feet (4 patients) in which the paralysis occurred between 18 months and 4 years after the commencement of antileprotic drug therapy in patients of LL-BL type leprosy who had chronic lepra reaction for which many supportive drugs had been tried. Two patients ( 3 feet affected) were taking both dapsone and thiambutosine at the time, but the others were on sulphones only at the time of the acute paralysis. In one patient the episode may have been precipitated by an infected foot and in another by physical exhaustion. Another patient was known to have treated himself on several occasions with prednisolone, causing acute withdrawal symptoms which may have predisposed to his paralysis. The 3 patients in whom both feet were affected fall into the second group, and a period of at least 10 months separated the two acute paralytic episodes.

There was no correlation between the time taken for recovery and the relation of paralysis to the commencement of drug therapy or the leprosy type. The first signs of recovery appeared as early as 1 month, although it was delayed as long as one year in several feet. Usually there was some return of function within 3 to 6 months. Functional recovery occurred as early as 2 months, but was delayed to 2 years in 2 feet, the average being 12 months. The earliest full recovery was at 8 months, but one patient showed slow improvement in power for 6 years.

All patients were provided with foot-drop springs, which appear to aid recovery; 9 patients ( 11 feet) were given bed rest, plaster of Paris gutter splints, and daily passive exercises. In 2 cases walking plaster of Paris casts were applied, but these procedures did not seem to influence the final outcome.

No patient received corticosteroids in the period immediately following the foot-drop. ACTH in small doses was given to 7 patients at the time of the foot drop. Stibophen injections were also given to these and other patients in the group. Other supportive drugs were prescribed for most of the patients, but it is difficult to discover any relation between the drugs given and the time taken for recovery.

Group l(b). This is the group of patients who developed some degree of muscle weakness and proceeded to functional recovery.

They included 22 males with 28 feet affected, and 4 females with 4 feet affected, 10 feet of LL type, 11 of BL, 9 of BB, 2 of BT. In most of these cases, the weakness was not complained of as the foot was still functional due to a tibialis anterior muscle power of over 3, or hyperaction of the toe extensors. The maximum duration of weakness, as stated, was 12 months.

All these patients were supplied with toe-raising springs, several had complete drop of the other foot at the same time and were hospitalized because of generalized reaction. Most of the patients were taking dapsone, but to some, thiambutosine was given once the foot weakness had occurred. It is not easy to define the time of occurrence, but 5 patients ( 6 feet) showed the loss of muscle power within the first 6 months of treatment and definite improvement occurred within the next 6 months; 4 patients ( 5 feet) showed continued slow loss over a period of up to 12 months, but later showed recovery.

From this study of these 51 feet in Group 1, it is obvious that acute muscle weakness is most common with the BT-BB-BL type of leprosy although the LL group formed the highest proportion of the cases in the whole group under 
review. Also, the paralysis is most likely to occur prior to, or within, 6 months of starting antileprotic therapy.

Group 2(a). There were 19 patients with 23 feet showing complete foot drop on admission. The duration of paralysis was at least 9 months with a maximum of 10 years.

The group includes 13 left feet and 10 right feet, 16 males ( 19 feet) and 3 females ( 4 feet). Leprosy types of the 23 feet were 4 of LL, 5 of $\mathrm{BL}, 12$ of $\mathrm{BB}, 2$ of BT. Age range 12 to 58 years. All of these patients underwent surgery for foot drop correction; some were skin-smear-negative on admission to Hay Ling Chau, and 4 were admitted for treatment of foot problems.

Group 2(b) consisted of 3 patients who had only foot weakness on admission, but later required surgery for foot-drop correction.

(i) A male of 14 years (no. 1655) of BL type of leprosy. On admission he gave a history of 18 months leprosy with right foot weakness for 12 months. Further acute loss occurred during up-grading lepra reaction while on dapsone therapy $(100 \mathrm{mg}$ weekly). In spite of a full-length walking plaster cast for 6 weeks, no recovery occurred. Surgery was performed 11 months after the onset of the acute paralysis.

(ii) A female, aged 49 years (no. 1611), with BT type leprosy of many years' duration and already BI negative, was admitted with weakness of the right foot and paralysis with infection of the left foot associated with bone disintegration. No attempt was made to support the right foot or give physiotherapy while she was on bed rest for the left foot. A complete right foot drop which may have been aggravated by disuse atrophy occurred. This patient was receiving dapsone.

(iii) A female patient, aged 38 years (no. 1726), with BL type leprosy of 3 to 4 years' duration. The right foot was weak on admission and all superficial nerves were tender. She was given small doses of sulphones because of recurrent reaction of the upgrading type, with rapid fall in BI. She developed complete foot drop in both feet 7 months after admission. At that time she was not receiving any antileprotic drug and was suffering from hepatitis. She had been given a 3-week course of prednisolone 3 months earlier for acute ulnar neuritis. The patient was given toe-raising springs for use by day and gutter splints at night and was admitted to hospital. Low dosage dapsone was resumed 2 months later. The left foot showed good recovery. The right foot showed no sign of recovery for 12 months, but then tibialis anterior muscle strength returned to Grade 2 only. Surgery for right foot-drop correction was performed 27 months after the foot-drop had occurred.

Group 2(c). This consisted of 3 patients (5 feet affected) in whom muscle weakness was present; surgery was performed early in their course of therapy.

(i) A male of 43 years (no. 1705) with BB type leprosy. He had a history of right foot weakness for 1 year prior to admission. Nine months af ter admission a complete foot-drop occurred, the patient suffered much reaction and only received small doses of dapsone and Sulphetrone over this period. Twelve months after the foot-drop the tibialis anterior muscle showed strength 2 ; after a further 6 months, without further improvement, operation for foot-drop correction was performed.

(ii) A male aged 21 years (no. 1695) with LL type leprosy. On admission the feet muscles were apparently of normal strength, but during the following year he showed slight weakness of both tibialis anterior muscles (strength 4). During the next 10 months the right foot had further loss of power down to 1 and a 
foot-drop correction operation was performed on both feet over the next 9 months, although the left tibialis anterior was then strength 4 . Surgery was probably performed as the patient was already BI negative and asking for discharge, and unlikely to continue foot care at home. The patient had received dapsone and Sulphetrone in small doses for the first year during which the loss occurred, followed by thiambutosine during which time some recovery of power in the left foot was noted.

(iii) A male of 14 years (no. 1656) with BB type leprosy. On admission, a voluntary-muscle test showed left ulnar paralysis and tibialis anterior muscle power of 4 in the right foot and 2 in the left foot. The duration of weakness was unknown, but over the following 5 months little change was noted and foot-drop correction surgery was carried out on both feet, in the hope of enabling him to return to school soon. The patient was receiving thiambutosine. Several years later he developed ulnar paralysis of the other hand.

It would appear in the light of this study that these 5 feet might have shown more recovery had surgery been delayed, and might eventually not have required surgery at all.

Group $2(d)$ consisted of 6 patients ( 8 feet affected) in whom surgery would probably have been advisable but was not performed for various reasons; the group included 5 males (6 feet) and 1 female ( 2 feet); 3 patients ( 5 feet) with LL type leprosy, 2 of BL type, and 1 of BT type. Three of these patients refused surgery and 2 of them absconded before they were fit for surgery. One died from intercurrent disease.

\section{Discussion}

In summarizing the above, it is interesting to note that there were only 7 patients with a total of 9 feet who, while under treatment, developed paralysis to a degree where surgery was recommended. Of this group, 3 patients (5 feet) might in fact have recovered if they had been observed for a longer period, while 2 patients ( 2 feet) refused surgery. Of the feet with marked or complete paresis in Group 1, which recovered all or some power, there were 17 of the Borderline type and only 2 of the Lepromatous type. Those with partial muscle weakness included 22 of Borderline type leprosy and 10 of Lepromatous type. Hence out of the 400 feet examined, 39 of the Borderline type and 12 of the Lepromatous type developed motor nerve deficit during treatment, but proceeded to recovery of functional power. This represents $17.5 \%$ of the Borderline and $7 \%$ of the Lepromatous-type feet surveyed.

Of the feet in Group 2, with complete paralysis, usually of longer duration, there were 28 of Borderline and 11 of Lepromatous type. This represents $12.5 \%$ of the Borderline and $7 \%$ of the Lepromatous feet in the whole group.

Therefore, a total of $14 \%$ of the Lepromatous feet and $30 \%$ of the Borderline feet surveyed were affected with some clinically detectable degree of muscle weakness or paralysis.

Hence it is obvious that patients with the unstable BL-BB-BT forms of leprosy are the most likely to develop acute paralysis, and this is of ten in association with reaction. A large proportion of these acute neuropathies will occur before, or in the early stages of, antileprotic therapy. But the majority of patients who commence therapy within a few months of acute paralysis can expect functional recovery. It is noticed that, especially in the $\mathrm{BB}$ group of patients this acute 
episode frequently occurs within 3 to 6 months of commencing antileprosy drug therapy, but may also be precipitated by intercurrent diseases or even inoculations such as anti-cholera, smallpox and T.A.B. vaccine. Although the use of a toe-raising spring is accepted as aiding recovery, other forms of splinting or complete immobilization do not seem to make much difference in the mobile patient. If, however, the patient is confined to bed for some reason. the use of a gutter splint, with the ankle held at $90^{\circ}$ to prevent contracture of the tendo Achilles, is advisable. This also prevents development of fixed inversion of the foot due to muscle imbalance.

\section{Development of New Treatment Regimes}

This incidence of paralysis seemed high, especially the proportion of patients who developed a neural deficit after commencing antileprotic treatment. Various different drug regimes were tried, especially when other workers confirmed the impression that nerve lesions were less common in Borderline patients treated with drugs other than dapsone, than occurred in those treated with any of the sulphones. Karat (1966) states that Borderline-type patients have a particular predilection to develop acute multiple paralyses while taking dapsone.

\section{Second Group for Review}

A review of more recent patients showed interesting contrasting results. The selected group was 100 consecutive admissions who were admitted between September 1970, and January 1972. Of these. 37 (10 women and 27 men) were admitted for reconstructive surgery, and for those patients a total of 25 foot-drop corrections were performed. The remaining 63 patients included:

$\begin{array}{lcccc} & \text { Male } & \text { Female } & \text { Total } & \text { \% of active patients } \\ \text { Lepromatous (LL) } & 19 & 4 & 23 & 36 \\ \text { BL } & 16 & 4 & 20 & 32 \\ \text { Borderline (BB) } & 8 & 4 & 12 & 19 \\ \text { BT } & 7 & 1 & 8 & 13\end{array}$

The practice had developed that any patient who was Borderline in type and had tender nerves would begin treatment with thiambutosine. These borderline patients included those near lepromatous (BL and LI) unless they were relapsed cases or were suspected of having dapsone resistant organisms and it was desirable to test clinically for dapsone resistance as laboratory tests are difficult to arrange.

Following this practice, 32 of the 40 patients in this BL-BB-BT group were started on treatment with thiambutosine, 7 continued dapsone, and one commenced with Lamprene. The routine now is to give thiambutosine in full dosage ( $1 \mathrm{~g}$ weekly) by intramuscular injection for a minimum of 3 months or until all signs of nerve lesions have been quiescent for 3 months, and then to add dapsone in an attempt to change over for maintenance.

Supplementary "anti-reactive therapy" is also given. Largactil $50 \mathrm{mg}$ one to three times daily according to need, Phenargan $25 \mathrm{mg}$ at night, and chloroquine $400 \mathrm{mg}$ at night together with vitamins and calcium routinely, and supplements of iron as indicated. Stibophen is also given for more acute episodes, and sometimes 20 units of ACTH. Corticosteroids are reserved for the really acute episodes, especially paralysis occurring in the BB-BT-TT group, but they are now used more frequently for this group than previously. 
Patients in reaction may continue thiambutosine for 15 to 18 months; if, after that time, the $\mathrm{BI}$ is still high and they cannot tolerate dapsone without reaction, they are advised to take Lamprene. These are usually the patients in the BL-LL category in whom the $\mathrm{BI}$ fall is slow. The aim to change over by 18 months is because of the fear of thiambutosine resistance, which has been proved already in a number of our patients.

Of this group of 40 patients, a total of 24 had definite neural deficits on admission. In all but 2 of the patients taking thiambutosine this deficit remained stationary or diminished while on thiambutosine, and in 10 some clinical recovery occurred. In some cases this was functional recovery. Definite improvement was noted in 9 patients who were admitted with recent deficit: in 5 of these a 3-week course of prednisolone was given as the paralysis had occurred within the 2 months previous to the start of intramuscular thiambutosine therapy. In the other 4, no corticosteroids were used since the paralysis was of longer duration. But all showed definite clinical improvement, usually proportional to the duration of the paralysis.

One male patient (no. 2161) developed bilateral acute foot-drop 3 months after commencing intramuscular thiambutosine and was given a 3-week course of corticosteroids. Recovery was first evident within one month, functionally adequate within 4 months, and complete within 18 months. The patient had a triple palsy of the left arm which had been present for many years, but the condition of this remained unchanged.

One exception was patient no. 2177 , a male aged 30 with BL type leprosy, who absconded twice from the institution, so that therapy was irregular. He also attempted suicide on several occasions. A few weeks after one suicidal attempt, when he ingested some unidentified drug and was admitted to hospital in a coma he developed an acute paralysis of both feet which had already been weak on admission. Since the patient had to be transferred to a psychiatric hospital a few weeks later no adequate follow-up is available.

Another exception was patient no. 2121 , a male aged 53 years, who suffered an acute paralysis of one leg 3 months after commencing thiambutosine. The patient died 6 weeks later from cerebral syphilis.

Of the other patients in the BL-BB-BT group with neural deficit there was no deterioration clinically while they were taking thiambutosine, but several did complain of neurological symptoms when dapsone was added to the thiambutosine. In these cases, dapsone was stopped and thiambutosine alone given for a further 3 months, usually with rapid cessation of the nerve symptoms. In some of these patients there was apparently reduction in the size and degree of areas of anaesthesia. The patient who began with Lamprene had considerable involvement of his hands on admission with tender nerves and much E.N.L. No previous antileprotic therapy had been given. He was apparently of the down-grading BL-LL type and was elderly. He developed foot drop 3 months after commencement of treatment, but proceeded to complete recovery within 9 months and also showed some improvement in the power of his hands.

Of the 7 patients taking dapsone, one was BT type who had previously had dapsone without ill effect and had evidence of nerve damage in the skin lesions only. The other 6 on dapsone were BL type but mostly tending to LL. Of the 2 who showed increased loss of nerve function, one was patient no. 2103 who on admission had had a left claw hand for 8 months and continued to show slow loss of right ulnar and median function over the following 12 months. The other was 
patient no. 2107 who had been receiving irregular treatment for 5 or 6 years and was suspected of showing resistance to both dapsone and thiambutosine. On admission he was given dapsone by injection, when he already showed some weakness of the right foot and both hands; 6 months later he suffered a complete foot drop. Prednisolone was given and the patient then agreed to take Lamprene. We assume by his lack of clinical response that he is now resistant to dapsone. Twelve months later the foot showed no recovery.

In this group the incidence of increasing neural deficit in patients receiving thiambutosine was 3 patients ( 5 feet) out of 32 patients in the groups considered at risk, as shown by the first survey. This represents $7 \%$ of the feet at risk, which compares favourably with the incidences of $17.5 \%$ and $30 \%$ for the two classifications in the previously surveyed group. It is interesting to note that of these 3 patients the only one with a reasonable follow-up regained full use of his feet within 18 months. No patient in the group who received thiambutosine as initial therapy, who was admitted with functional feet and received the full routine of medical and physiotherapy care, has required a foot-drop correction.

The other interesting point is that some of the BL-LL patients were initially treated with thiambutosine with good progress until attempts to change to dapsone were made. In a number of cases this was rapidly followed by neurological symptoms which abated on withdrawal of the dapsone and continuation of thiambutosine.

\section{Discussion}

The mode of action of thiambutosine has not been fully determined, but in Chinese patients at least it does seem to spare nerve function, especially in the initial stages of treatment, as suggested by Karat (1966).

In the first group studied, the incidence of acute paralysis in the unstable Borderline patients was high in the first 6 months of treatment, and this rate can apparently be lowered by the use of thiambutosine as the initial drug for antileprotic therapy assisted by corticosteroids for 3 weeks in the BB-BT-TT patients when acute paralysis does occur. However, by the first study it is obvious that spontaneous recovery of nerve function does occur in a large proportion of these patients, provided they are given antileprotic drugs and adequate supportive treatment in the form of physiotherapy, splints and any other necessary medication.

Even in simple clinics where sophisticated treatment is not possible, recovery should occur in a large proportion of the patients who are seen early after paralysis has occurred. Where such treatment as thiambutosine is available it should be possible to achieve functional recovery in almost $100 \%$ of Borderline patients seen within one month of the onset of the acute paralysis. This is very encouraging, and with proper application could result in a definite reduction in the number of patients with permanent disability.

\section{Conclusions}

(1) Acute paralysis in Borderline-type leprosy tends to spontaneous recovery.

(2) Recovery can be assisted by supportive therapy, especially antileprotic therapy, physiotherapy and use of active splints. 
(3) Thiambutosine as the drug of choice reduces the incidence of severity of acute nerve lesions in these patients and appears to aid recovery.

(4) Full-length plaster casts or. splints do not appear to assist recovery, providing the patient is mobile and using a toe-raising spring, but they have a definite place in preventing deformity if the patient is confined to bed or is uncooperative.

(5) Corticosteroids appear to assist recovery if given within the first 2 months of an acute paralysis. A 3 -week course seems adequate; it is not necessary to continue until recovery is complete.

(6) Earliest signs of recovery may be detected within a few weeks of the initial paralysis, but may be delayed for up to 12 months and occasionally even longer. Increasing muscle power has been observed for up to 6 years after an acute paralysis, but usually has reached its maximum strength within 2 years.

(7) In foot-drop the beginning of antileprotic therapy even 6 months after the acute loss, with the use of a toe-raising spring, has resulted in return of muscle power.

(8) Surgical foot-drop correction should not usually be considered until at least 6 months of treatment has been given after an acute paralysis, and should if possible and practical be postponed for even longer if there is definite evidence of returning muscle power.

\section{References}

Fritschi, E. P. (1971). Reconstructive Surgery in Leprosy, p. 142. John Wright and Sons Ltd., Bristol.

Furness, M. A. (1972). Physical therapy in the management of recent paralysis in leprosy. Rehabilitation in Asia. Vol. 8, No. 1, pp. 18-21.

Goodwin, C. S. (1968). The use of the voluntary muscle test in leprosy neuritis. Lepr. Rev. 39 , 209-216.

Karat, A. B. A. (1966). Recent advances in leprosy work. J. Christian Med. Ass. India, Dec. 1966.

Price, E. W. (1964). The care of the feet. In Leprosy in Theory and Practice, 2nd Edition, p. 524. Bristol: John Wright \& Sons.

Ridley, D. S. and Jopling, W. A. (1962). A classification of leprosy for research purposes. Lepr. Rev. $33(2), 119-128$.

Warren, A. G. (1970). A Foot Drop Spring. Lepr. Rev. 41, 100-102. 